\title{
Identification of Human-Derived Attractants to Simulium damnosum Sensu Stricto in the Madi-Mid North Onchocerciasis Focus of Uganda
}

\author{
Devon Cozart, ${ }^{1}$ Thomson Lakwo, ${ }^{2}$ Canhui Liu, ${ }^{1}$ Denis Loum, ${ }^{3}$ Benjamin Jacob, ${ }^{4}$ Eddie W. Cupp, ${ }^{1}$ and Thomas R. Unnasch ${ }^{1 *}$ \\ ${ }^{1}$ Center for Global Health Infectious Disease Research, University of South Florida, Tampa, Florida; ${ }^{2}$ Vector Control Division, Ministry of Health, \\ Kampala, Uganda; ${ }^{3}$ Nwoya District Local Government, Nwoya, Uganda; ${ }^{4}$ College of Public Health, University of South Florida, Tampa, Florida
}

\begin{abstract}
Human landing collections (HLCs) have been the standard method for the collection of black flies that serve as vectors for Onchocerca volvulus, the causative agent of onchocerciasis or river blindness. However, HLCs are inefficient and may expose collectors to vector-borne pathogens. The Esperanza window trap (EWT) has been shown to be a potential alternative to HLCs for the collection of Simulium damnosum, the principal vector of O. volvulus in Africa. To improve the performance of the EWT, sweat from individuals highly attractive or less attractive to $S$. damnosum sensu stricto was examined by gas chromatography and mass spectroscopy. Twelve compounds were identified which were solely present or present in increased amounts in the sweat of the highly attractive individuals. Two of these compounds (naphthalene and tert-hexadecyl mercaptan) were found to be attractive to S. damnosum s.s. in behavioral assays. Traps baited with these compounds outperformed those baited with the current standard bait of worn socks. Using these newly identified compounds as baits will make the EWT more efficient in collecting vector black flies and may enhance the potential utility of the EWT as a local vector control measure.
\end{abstract}

\section{INTRODUCTION}

Onchocerciasis is a parasitic infection caused by the filarial nematode Onchocerca volvulus. The parasite is transmitted through the bites of black flies of the genus Simulium that develop as larvae in fast running rivers and streams; hence, the disease is commonly called "river blindness."1 Currently, 217.5 million people live in areas that are known to be endemic for onchocerciasis, and more than $99 \%$ of these cases are concentrated in 28 countries in sub-Saharan Africa. ${ }^{2}$ Mass drug administration (MDA) with ivermectin has been the main tool in the fight against onchocerciasis in both the Americas and Africa. ${ }^{3}$ The proof of principle by Diawara et al. ${ }^{4}$ that ivermectin MDA alone can interrupt the transmission of $O$. volvulus led to a paradigm shift from control to elimination in Africa. ${ }^{5}$

The major criterion contained in the 2016 WHO guidelines for the verification of onchocerciasis elimination is surveillance of the vector population. ${ }^{6}$ The standard method used in the collection of the vector (mainly Simulium damnosum sensu lato) is human landing collections (HLCs). ${ }^{7}$ However, HLCs are inefficient and may increase the risk exposure of the collectors to O. volvulus. The Esperanza window trap (EWT), developed in Mexico and subsequently evaluated in Burkina Faso and Uganda, has been found to be an effective replacement for HLCs for S. damnosum..$^{8-12}$ In the initial trials of the EWT, sweat-impregnated articles of clothing (trousers and socks) were used in conjunction with $\mathrm{CO}_{2}$ as baits for the EWT. ${ }^{8,9}$ In an attempt to develop a more consistent and attractive bait, an analysis of compounds that were common to sweat collected from three individuals were identified. ${ }^{13}$ These compounds, as well as several others that had been shown to be attractive to other species of hematophagous insects, were evaluated for their ability to attract $S$. damnosum sensu stricto in Burkina Faso, using a combination of physiological (electroantennogram) and behavioral ( $Y$-tube) assays. ${ }^{13}$ Five attractive compounds were identified in this manner. Plastic aroma beads

*Address correspondence to Thomas R. Unnasch, Global Health Infectious Disease Program, 3720 Spectrum Blvd., Suite 304, Tampa, FL 33612. E-mail: tunnasch@usf.edu infused with these compounds were found to be as effective, but not more effective than worn socks when used as baits in conjunction with $\mathrm{CO}_{2}$ on the EWT. ${ }^{13}$ This was perhaps not surprising, as this study focused on compounds that were found in all sweat samples analyzed. However, it has long been known that individuals differ greatly in their attractiveness to other hematophagous insects. ${ }^{14}$ By identifying differences in the composition of sweat collected from highly attractive versus less attractive individuals, it might be possible to develop a bait formulation that would be more highly attractive than one that incorporated compounds that were common to the sweat of all individuals. Here, we have tested this hypothesis, comparing the composition of sweat collected from individuals who were more attractive to S. damnosum s.s. with the sweat of individuals of average or less than average attractiveness. The compounds that were unique to or enhanced in the sweat of the highly attractive individuals were then evaluated for their attractiveness to $S$. damnosum s.s. using Y-tube and field-based assays.

\section{MATERIALS AND METHODS}

Study sites. Studies were carried out in Gonycogo and Laminatoo villages in the Koch Goma subcounty of the Nwoya district of Northern Uganda. Details regarding these communities are provided in an earlier publication. ${ }^{11}$ Both communities are located along the Ayago River. In previous studies, the sibling species of $S$. damnosum s.l. present in these communities was identified as $S$. damnosum s.s. ${ }^{11}$

Assaying the relative attractiveness of different individuals. A total of 16 individuals (eight each from Gonycogo and Laminatoo) were recruited to serve as vector collectors at the beginning of the study. The vector collectors were assigned to one of eight collection locations situated within a 500-m radius of the major $S$. damnosum s.s. breeding site located near each community. The collectors were trained in standard black fly collection procedures, ${ }^{15}$ and collections were begun on May 15, 2017. Collections began at sunrise (ca 7 AM) and continued until sunset (ca 6 PM). On the second day of collections, the collectors were rotated to a different 
collection site, and collections were conducted as before. Collections continued approximately twice per week (interrupted occasionally by inclement weather or important community events and holidays) through August 11, 2017, encompassing 24 total collection days. All collectors thus spent 3 days at each collection site, to control for differences in fly density among the collection sites.

The total number of flies collected by each individual in each community was normalized by dividing the total number of flies collected by each individual by the total number of flies collected over the course of the study. This was performed to adjust for the difference in the overall fly density between the two communities.

Collection of sweat samples. Sweat samples were collected from the two most attractive individuals, the two least attractive individuals, and two individuals exhibiting average attractiveness. To collect the sweat samples, each of the individuals enrolled in the study were provided with a new cotton tee shirt, a new pair of cotton underpants, and a new pair of white cotton socks. The shirts, underpants, and socks were laundered three times in plain water before use. Each collector wore the clothing continuously for 72 hours. The worn garments were then collected, placed in ziplock polyethylene bags (one bag per individual, containing the collected shirt, underpants, and socks), and transported to the United States. The clothing samples were stored at $-20^{\circ} \mathrm{C}$ in the sealed bags until analyzed.

Analysis of sweat samples. Sweat samples were analyzed by coupled gas chromatography-mass spectroscopy (GC-MS) as previously described. ${ }^{13}$ In brief, a Solid Phase Microextraction - Overcoated (SPME-OC) fiber assembly $65 \mu \mathrm{m}$ PDMS/DVB (Cat \#57439-U, Sigma-Aldrich, St. Louis, $\mathrm{MO}$ ) was used to detect the compounds in the worn clothes. $A$ fiber was put into the bag containing the dirty clothes for 24 hours at room temperature to absorb volatile compounds. The SPME-OC fibers were analyzed on an Agilent 7200 GC-QToF (Agilent, Inc., Santa Clara, CA). The sweat components were thermally desorbed in the $\mathrm{GC}$ inlet at $320^{\circ} \mathrm{C}$, and the individual components were resolved on a HP-5ms capillary column (Hewlett Packard, Palo Alto, CA) (30 m, i.d. 0.25 mm) using helium as the carrier gas at a flow rate of $1.2 \mathrm{~mL} /$ minute. The column was heated from $60^{\circ} \mathrm{C}$ to a final temperature of $320^{\circ} \mathrm{C}$ at a rate of $20^{\circ} \mathrm{C} /$ minute. Compounds were ionized using electron ionization for initial identification. Chemical ionization was used to confirm molecular ions using methane as the reagent gas and both positive and negative modes (40-110 eV, compound dependent) to obtain optimum signal-to-noise ratio. The total ion chromatographs were de-convoluted using Agilent's deconvolution algorithm, and the resulting fragmentation patterns were imported into Mass Profiler Professional for statistical analysis. Datasets, prepared for each time point and from both SPME fiber materials, were combined to create an entity list for each subject containing all compounds present in their sweat. Spectra of entities identified as present in the sweat of both highly attractive individuals but absent or in very reduced amounts in the sample from the low and average attractive individuals were compared with the fragmentation patterns contained in the NIST/Wiley mass spectra library for tentative identification (Wiley Interscience, Hoboken, NJ), as previously described. ${ }^{13}$

Y-tube assays. Host-seeking $S$. damnosum s.s. females were collected by human landing collectors in Gonycogo and Laminatoo. The flies were captured alive into glass tubes which were then wrapped in a moist towel soaked in water and put in a cool box for transportation to the laboratory. Flies were confirmed as S. damnosum s.s. using morphological characters before being used in the $Y$-tube assays. All flies were used in the $Y$-tube assays within 24 hours of capture.

Y-tube assays were conducted in a darkened climatecontrolled room located at the Molecular Laboratory of Gulu University, essentially as previously described. ${ }^{13}$ Air flowing through the chamber was passed through a charcoal filter to remove volatiles in the ambient air. Twenty flies were relocated into the closed release chamber at the trunk of the Y-tube and allowed to acclimate for 10 minutes. During this time, samples were prepared on filter papers which had previously been rinsed in hexane and dried. A total of $20 \mu \mathrm{L}$ of the sample compound dissolved at various concentrations in hexane was pipeted onto the filter paper, and $20 \mu \mathrm{L}$ of hexane was pipeted onto a second filter paper, which served as the control. After the acclimation period, the filter papers were loaded into separate end chambers, and the flies were released and allowed to roam freely in the apparatus for 20 minutes. All chambers were then closed, and the number of flies in either arm of the Y-tube apparatus, those remaining in the trunk of the apparatus, and those still in the release chamber, were counted. The compounds were initially tested at 1:100 and 1: $1,000(\mathrm{w} / \mathrm{v})$ dilutions. Those that appeared to exhibit some activity at these dilutions were then tested over a wider range of concentrations. Each concentration of each compound was tested in six independent trials. The arms containing the control and compound samples were interchanged on every trial to eliminate potential position effects. The Y-tube was thoroughly cleaned with ethanol between runs, and control runs were carried out between each experimental trial to ensure that no residual attractant remained in the apparatus. Data were analyzed using a likelihood ratio test $(\alpha=0.05)$, as previously described. ${ }^{13}$

Field evaluation of compounds as baits. Naphthalene and tert-hexadecyl mercaptan were dissolved in mineral oil at a $1 / 100$ concentration $(w / v)$. This solution was then used to prepare two 10-fold dilutions in mineral oil. Aliquots of $25 \mathrm{~g}$ of plastic aroma beads (Bitter Creek Candle Supply [www. candlesupply.com]) were placed into a series of $50 \mathrm{~mL}$ conical centrifuge tubes, and the tubes were filled with the solutions of the compounds. The tubes were placed on a tube rotator, and the beads were allowed to absorb the solutions for 2 days. The excess solution was decanted from the beads, and the tubes were sealed and transported to the field sites.

Four EWTs containing a thin black stripe (the optimal design for the collection of $S$. damnosum s.s. in Uganda ${ }^{16}$ ) were set up at locations near the larval breeding sites at Gonycogo and Laminatoo. The trap sites were chosen so that they were within $500 \mathrm{~m}$ of the breeding site but separated from each other by at least $50 \mathrm{~m}$. All traps were baited with 5 - $L$ containers of yeast and sugar dissolved in water to create $\mathrm{CO}_{2}$, as previously described. ${ }^{13}$ Baits containing aroma beads impregnated with different concentrations of naphthalene and tert-hexadecyl mercaptan were prepared by placing approximately $20 \mathrm{~g}$ of the beads into a woman's nylon stocking and the top of the stocking tied off. The baits (and worn socks as the control) were attached to the top of the trap by tying them onto the frame with a string.

The traps were allowed to collect flies from sunrise until sunset. The insects collected by the trap were then removed 


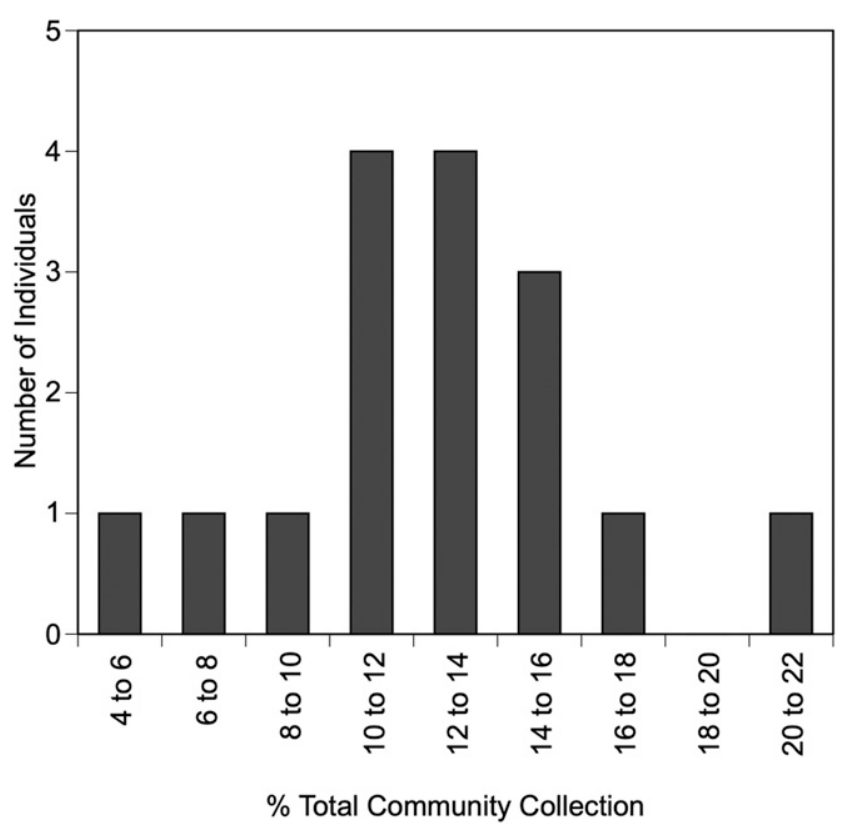

FIGURE 1. Mean percentage of the community daily catch collected by human landing collectors. The $x$ axis represents the percentage of the mean community collection caught by an individual and the $y$ axis the number of individuals collecting this percentage.

and the S. damnosum s.s. identified by morphological keys. On the second day of the trial, baits were rotated among the traps to control for position effects. Each trial was continued for a total of 8 days so that each bait was evaluated twice at each trap location. The yeast solution was replenished daily, and the baits were replaced every other day. The number of flies collected each day by each bait was normalized to the total number of flies collected by all traps at the community during the day, controlling for differences in the fly densities from day to day (resulting from daily changes in weather conditions) and for the differences in fly densities in the two communities.

The proportional data were analyzed for normality using the Shapiro-Wilk test. Where normality assumptions were not violated, the data were analyzed using an Analysis of Variance (ANOVA) with a Tukey post hoc. In cases where normality assumptions were considered violated, the data were analyzed using the Kruskal-Wallis test with a Dwass, Steel, Critchlow-Fligner (DSCF) post hoc. Significance was tested at an $\alpha=0.05$ level.

Ethical statement. The experiments included in this study were reviewed and cleared by the Institutional Review Committee of Vector Control Division (VCD-REC/071), and final approval was granted by the Uganda National Council for Science and Technology (HS 2154). Approval was also obtained from the University of South Florida Institutional Review Board for Human Subject Research (protocol number CR5_Pro00015108). All individuals involved in the study provided written informed consent to participate.

\section{RESULTS}

To identify sweat volatiles specific to highly attractive individuals, it was first necessary to identify individuals who were more or less attractive to $S$. damnosum s.s. To accomplish this, human landing collectors were recruited in the two study
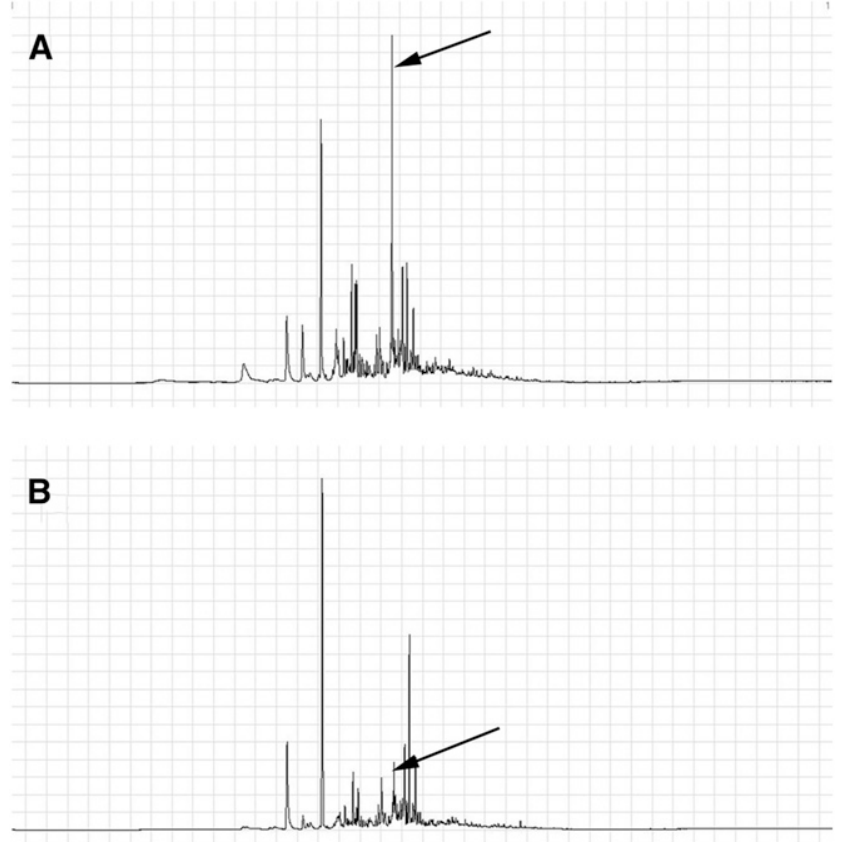

FIGURE 2. Gas chromatogram of sweat samples from one highly attractive and one less than average attractive individual. (A) Chromatogram from a highly attractive individual. (B) Chromatogram from an individual with less than average attractiveness. The arrow indicates the peak identified as 6,10-dimethyl-5,9-undecadien-2-one, which represented the major component of the sweat sample from the highly attractive individual but was a minor component in the sweat of the less attractive individual.

communities of Gonycogo and Laminatoo. Eight collectors were recruited in each community. The collectors were then assigned to eight separate collection sites located near the principal breeding site at each community, as described in Materials and Methods. Fly collections were carried out roughly twice per week, and the collectors were rotated daily among the collection sites. The collections continued for 24 days, so that each collector collected at each site three times, to control for differences in fly density among the different sites. The proportion of all the flies collected in the community by each individual was then calculated. The proportion of flies collected by each individual appeared to follow a normal distribution, ranging from $4.1 \%$ of the total community collection to $23.0 \%$ of the total community collection (Figure 1). Two individuals collected more than the mean proportion of each community collection plus one SD (16.9\%), whereas two individuals collected less than the mean community proportion minus one SD (8.1\%). The two most attractive individuals, the two least attractive individuals, and the two individuals with an average level of attractiveness were chosen for further analysis. These six individuals were provided with new cotton shirts, underpants, and socks and asked to wear them for 72 hours without removing them, while going about their normal activities. The clothing samples were then collected and the volatiles analyzed by GC-MS, as described in Materials and Methods. The individual traces were compared with one another to identify compounds that were unique to or present in greater amounts in the samples from the two highly attractive individuals but were absent or present in lesser amounts in the remaining samples (e.g., Figure 2). A total of 12 compounds were identified by this process 
TABLE 1

Compounds unique to or in enhanced amounts in sweat from highly attractive individuals

\begin{tabular}{lc}
\hline \multicolumn{1}{c}{ Compound } & Commercially available \\
\hline 1-Octanol & Yes \\
1-Pentadecanol & Yes \\
2-Butyl-1-octanol & Yes \\
2-Heptanone & Yes \\
6,10-Dimethyl-5,9-undecadien-2-one & Yes \\
Naphthalene & Yes \\
tert-Hexadecyl mercaptan & Yes \\
1-Heptatriacotanol & No \\
2-Methyl-1-dodecanol & No \\
4-Ethyloctane & No \\
4,8-Dimethyl undecane & No \\
Pentadecanal & No \\
\hline
\end{tabular}

(Table 1). Of these, seven were found to be commercially available (Table 1).

The seven commercially available compounds were purchased and tested for their attractiveness to S. damnosum s.s. using a Y-tube assay, as described in Materials and Methods. The results are summarized in Table 2. Of the seven compounds tested, two (naphthalene and tert-hexadecyl mercaptan) were attractive to $S$. damnosum s.s. in the Y-tube assay. Naphthalene was found to exhibit significant attraction at both concentrations tested, whereas tert-hexadecyl mercaptan was attractive only at the lowest concentration tested (Table 2).

Aroma beads impregnated with different dilutions of the two compounds were used as baits in the EWT. The compounds were used as baits alone and in conjunction with worn socks. The number of flies caught by the traps baited with the compounds was compared with traps baited with worn socks alone as the current standard. Traps baited with beads infused with 1/100 dilutions of naphthalene and tert-hexadecyl mercaptan collected significantly more $S$. damnosum s.s. than traps baited with worn socks (Figures 3 and 4, panel A). Traps baited with a 1/1,000 dilution of naphthalene collected a similar number of flies as did traps baited with worn socks (Figure 3, panel A), whereas those baited with a 1/1,000 dilution of tert-hexadecyl mercaptan or $1 / 10,000$ dilutions of either compound performed significantly worse than did traps baited with worn socks alone (Figures 3 and 4, panel A). Similarly, traps baited with worn socks combined with 1/100 dilutions of either compound significantly outperformed traps baited with worn socks alone (Figures 3 and 4, panel B). However, the numbers of flies collected by traps baited with both socks and beads impregnated with $1 / 100$ dilutions of the compounds was not significantly different from the number of flies collected by traps baited with $1 / 100$ dilutions of the compounds alone (compare panel A with panel $B$ in Figures 3 and $4 ; P>0.2$.

\section{DISCUSSION}

The collection of host-seeking black flies for field studies and other forms of entomological surveillance has classically relied on human landing catches. ${ }^{15}$ However, HLCs involve significant logistical and ethical issues, including the risk for exposure of humans to $O$. volvulus infection. It is against this background that significant efforts have been ongoing to design an efficient bait formulation that could be used to attract black flies to artificial traps. In Africa, initial studies identified human sweat compounds that attract $S$. damnosum s.l., the major $O$. volvulus vector species group. ${ }^{13}$ The study reported here provides further insight into which compounds are attractive to $S$. damnosum s.s., identifying two attractive compounds derived from highly attractive individuals.

Tert-hexadecyl mercaptan elicited a strong response in the Y-tube bioassays; this was supported by its performance in the field as a bait, where aroma beads impregnated with a 1:100 dilution of this compound significantly outperformed worn socks, which have been the gold standard bait for the EWT. Furthermore, traps baited with a combination of worn socks and tert-hexadecyl mercaptan beads did not collect more flies than traps baited with the beads alone, suggesting that the beads alone were a very effective bait. Foot odor investigations indicate that the skin-foot microbiome and pathogens infecting the foot can produce a variety of volatile sulfurous compounds similar to tert-hexadecyl mercaptan. For example, infection with Micrococcus (=Kytococcus) sedantarius may cause pitted keratolysis, ${ }^{17}$ a fairly common chronic condition in the Tropics ${ }^{18}$ in which keratin-degrading exoenzymes (serine proteinases) cause pits by eroding callus of the stratum corneum. ${ }^{19}$ This pathology produces a malodor characterized by the presence of dimethyl disulfide, dimethyl trisulfide, and 2-methyl-I-methylthio propane. ${ }^{20}$ Micrococcus (=Kytococcus) sedantarius foot infection also has the ability to produce methanethiol, a component of "cheesy" foot odor. ${ }^{21}$ This suggests that studies focusing on the possible higher attractiveness of individuals with pitted keratolysis to S. damnosum s.l. may be of interest.

Our study also demonstrated that naphthalene at a 1:100 dilution exhibited performance as a bait that was similar to that seen with tert-hexadecyl mercaptan. Naphthalene has been

TABLE 2

Y-tube analysis of attractiveness of identified compounds

\begin{tabular}{|c|c|c|c|c|}
\hline Compound & Dilution & Bait, $\mu \pm S D$ & Control, $\mu \pm$ SD & $P$-value \\
\hline 2-Butyl-1-octanol & $1: 1,000$ & $5.75 \pm 2.22$ & $7.75 \pm 1.50$ & 0.275 \\
\hline 2-Heptanone & $1: 1,000$ & $5.75 \pm 3.10$ & $7.75 \pm 2.50$ & 0.275 \\
\hline 6,10-Dimethyl-5,9-undecadien-2-one & $1: 100$ & $9.25 \pm 4.27$ & $8.75 \pm 3.40$ & 0.814 \\
\hline 6,10-Dimethyl-5,9-undecadien-2-one & $1: 1,000$ & $5.25 \pm 3.20$ & $6.75 \pm 1.71$ & 0.386 \\
\hline tert-Hexadecyl Mercaptan & $1: 1,000$ & $8.00 \pm 2.94$ & $6.25 \pm 2.87$ & 0.353 \\
\hline tert-Hexadecyl Mercaptan & $1: 10,000$ & $10.75 \pm 2.22$ & $6.25 \pm 1.26$ & 0.028 \\
\hline Naphthalene & $1: 100$ & $12.75 \pm 3.59$ & $5.25 \pm 1.71$ & $<0.001$ \\
\hline Naphthalene & $1: 1,000$ & $11.50 \pm 2.38$ & $4.75 \pm 1.50$ & $<0.001$ \\
\hline 1-Octanol & $1: 100$ & $6.33 \pm 4.62$ & $7.00 \pm 5.20$ & 0.752 \\
\hline 1-Octanol & $1: 1,000$ & $6.67 \pm 2.08$ & $7.00 \pm 1.00$ & 0.876 \\
\hline 1-Pentadecanol & $1: 1,000$ & $8.75 \pm 1.89$ & $8.25 \pm 5.97$ & 0.808 \\
\hline 1-Pentadecanol & $1: 10,000$ & $7.25 \pm 2.22$ & $6.00 \pm 1.63$ & 0.492 \\
\hline
\end{tabular}




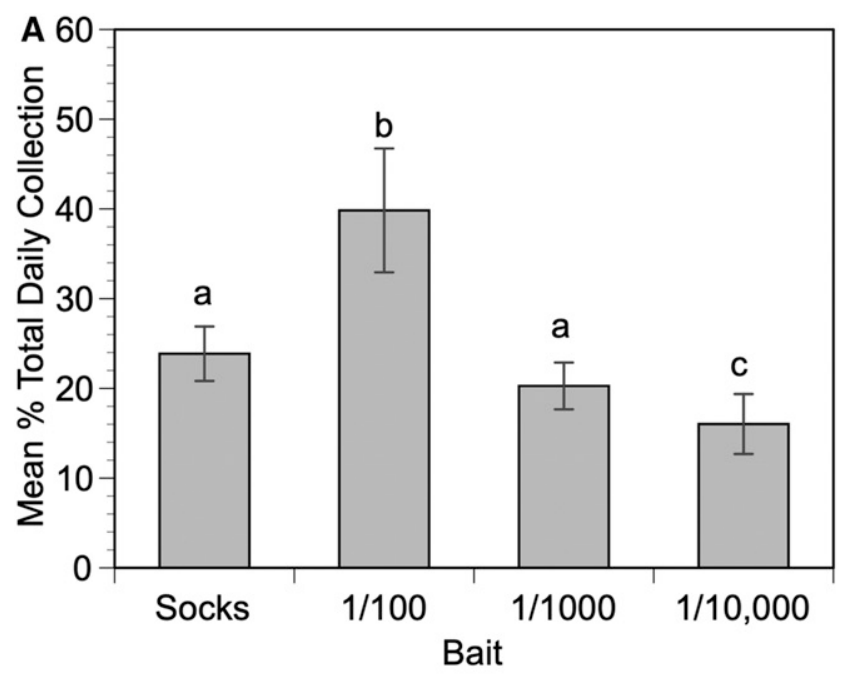

B

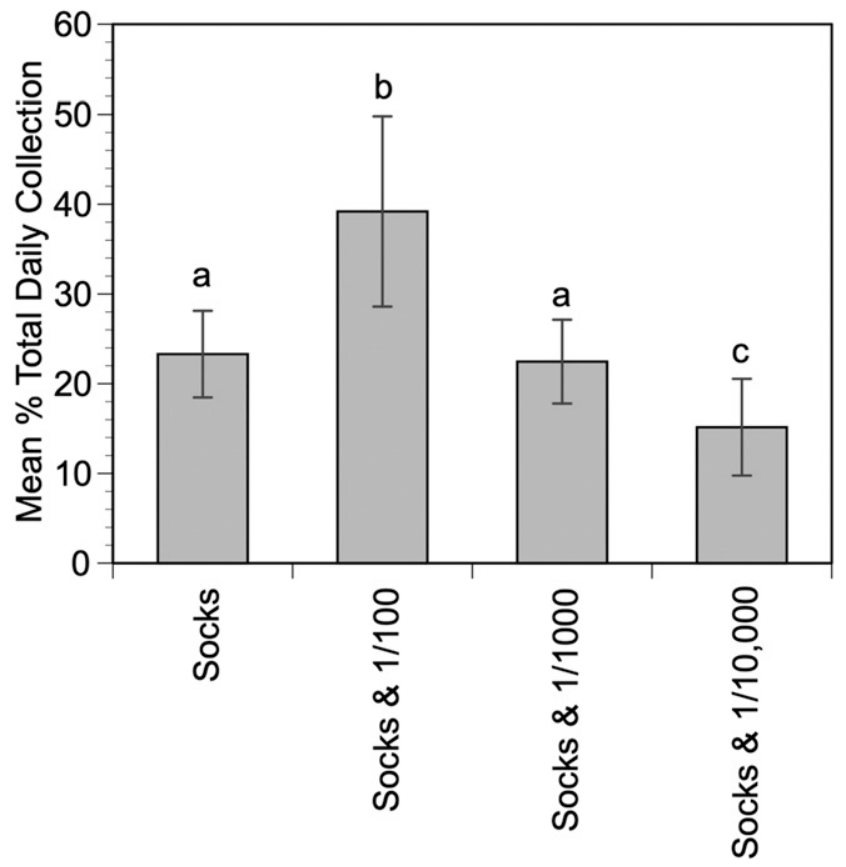

Bait

FigURE 3. Field trials of traps baited with aroma beads impregnated with naphthalene. (A) Trials conducted with beads alone compared with dirty socks. (B) Trials conducted with beads combined with dirty socks compared with dirty socks alone. Bars indicate the mean and error bars the SD of the proportion of the total daily collection in the community. Lower case letters indicate treatments where the proportion of the daily collection was not statistically significant from one another $(P>0.05)$.

reported to occur in humans,,$^{22}$ cattle, ${ }^{23}$ and dogs, ${ }^{24}$ and it could be involved in location of hosts for blood meals. Our results also support earlier laboratory observations by Verocai et al. ${ }^{25}$ that naphthalene was attractive to the North American black fly species Simulium vittatum in electroantennogram (EAG) and Y-tube assays. Both S. damnosum s.s. and $S$. vittatum are zoophilic species that may attack a variety of hosts. However, naphthalene was previously tested against five species of higher Diptera of veterinary importance and found to be largely repellant. ${ }^{23}$

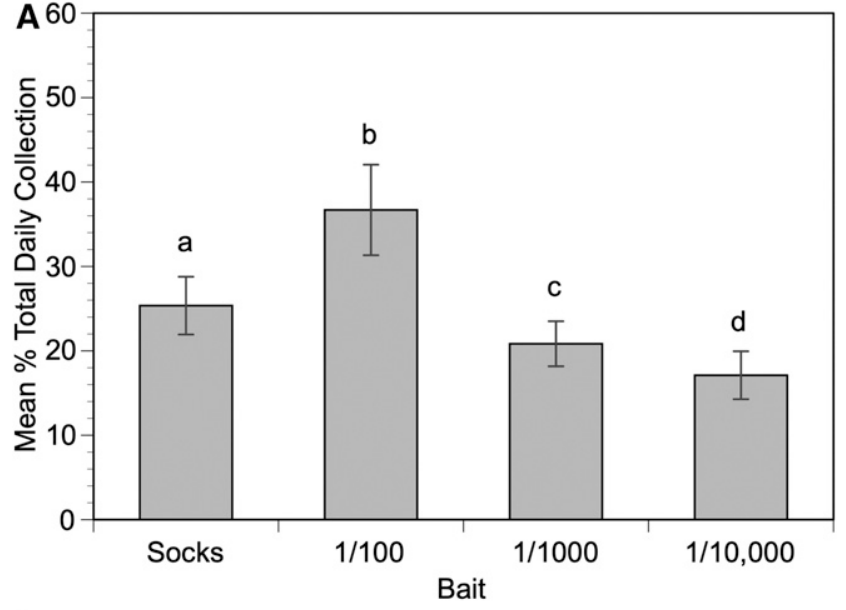

B

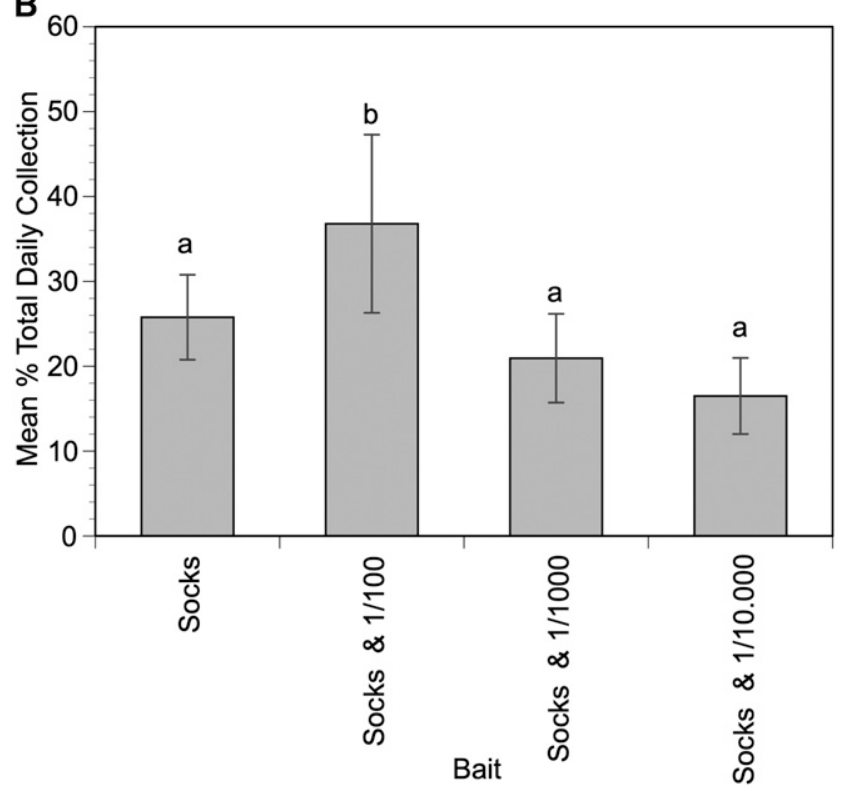

FIGURE 4. Field trials of traps baited with aroma beads impregnated with tert-hexadecyl mercaptan. (A) Trials conducted with beads alone compared with dirty socks. (B) Trials conducted with beads combined with dirty socks compared with dirty socks alone. Bars indicate the mean and error bars the SD of the proportion of the total daily collection in the community. Lower case letters indicate treatments where the proportion of the daily collection was not statistically significant from one another $(P>0.05)$.

The results described here provide useful information necessary to design a more efficient baited trap that could be used to attract large numbers of $S$. damnosum s.l. under field conditions. Onchocerciasis elimination programs in Africa have experienced challenges, ${ }^{26}$ and alternative strategies to accelerate elimination remain a priority. ${ }^{27}$ Studies conducted both in Mexico ${ }^{28}$ and Uganda ${ }^{16}$ have demonstrated that EWTs, when deployed in areas where people congregate, can significantly reduce biting by the Simulium vectors of $O$. volvulus, providing a supplemental strategy to MDA to accelerate reaching the goal of $O$. volvulus elimination. The EWT is inexpensive and simple to construct from materials that are commonly available in developing countries. ${ }^{16}$ It is quite possible that aroma bead baits prepared with terthexadecyl mercaptan and naphthalene may improve the effectiveness of EWTs, enhancing their potential as a local vector 
control measure. Aroma beads impregnated with these compounds are simple to prepare, and the ingredients are inexpensive. Although we have not yet explored the effective life of the baits, air fresheners prepared in the same way continue to be effective for weeks or months. An aroma bead bait for an EWT will cost $\$ 1.87$ (for $20 \mathrm{~g}$ of beads impregnated with naphthalene) and $\$ 2.99$ (for $20 \mathrm{~g}$ of beads impregnated with tert-hexadecyl mercaptan). Once the beads are prepared, they are stable when kept in a sealed container, are safe to handle, and can be transported at ambient temperatures; these characteristics would make their promotion and marketing much easier. In addition, traps baited with attractive lures can easily find an expanded market, being used for surveillance and evaluation of ivermectin MDA, the major criterion in the recent $\mathrm{WHO}$ verification guidelines. ${ }^{6}$ This expanded market may increase the availability and decrease the unit cost, both of the baits and the trap platforms.

\section{Received May 18, 2020. Accepted for publication June 24, 2020.}

Published online August 3, 2020.

Acknowledgments: We thank the management of Gulu University Administration, especially Richard Echodu, the head of the Molecular Biology Laboratory and dean of Faculty of Science, for making the necessary arrangements for the laboratory and allowing their staff to assist the research team. We thank the field staff in the Gonycogo and Laminatoo sites for their efforts in black fly collections and running the traps during the aroma bead evaluations. We are also indebted to the Carter Center for the timely disbursement of funds which allowed this study to be concluded successfully.

Financial support: This study was supported by a grant from the U.S. National Institute of Allergy and Infectious Diseases to T. L. and T. R. U. (Award \#1 R01 Al123245-01).

Authors' addresses: Devon Cozart, Canhui Liu, Eddie W. Cupp, and Thomas R. Unnasch, Center for Global Health Infectious Disease Research, University of South Florida, Tampa, FL, E-mails: devonc@ usf.edu, canhui@usf.edu, cuppedd@auburn.edu, and tunnasch@ usf.edu. Thomson Lakwo, Vector Control Division, Ministry of Health, Kampala, Uganda, E-mail: tlakwo@gmail.com. Denis Loum, Nwoya District Local Government, Nwoya, Uganda, E-mail: loumdenis2@gmail.com. Benjamin Jacob, College of Public Health, University of South Florida, Tampa, FL, E-mail: bjacob1@usf.edu.

\section{REFERENCES}

1. Katabarwa M, Lakwo T, Habomugisha $P$, Unnasch TR, Garms R, Hudson-Davis L, Byamukama E, Khainza A, Tukahebwa E, Richards FO, 2018. After seventy years of fighting an age-old scourge, onchocerciasis in Uganda, the end is in sight. Int Health 10: i79-i88.

2. World Health Organization, 2019. Progress report on the elimination of human onchocerciasis, 2018-2019. Wkly Epidemiol Rec 94: 513-524.

3. Thylefors B, Alleman MM, Twum-Danso NA, 2008. Operational lessons from 20 years of the mectizan donation program for the control of onchocerciasis. Trop Med Int Health 13: 689-696.

4. Diawara $L$ et al., 2009. Feasibility of onchocerciasis elimination with ivermectin treatment in endemic foci in Africa: first evidence from studies in Mali and Senegal. PLoS Negl Trop Dis 3: e497.

5. Mackenzie CD, Homeida MM, Hopkins AD, Lawrence JC, 2012. Elimination of onchocerciasis from Africa: possible? Trends Parasitol 28: 16-22.

6. World Health Organization, 2016. Onchocerciasis: Guidelines for Stopping Mass Drug Administration and Verifying Elimination of Human Onchocerciasis. WHO Document WHO/HTM/NTD/ PCT/2016.1. Geneva, Switzerland: WHO.

7. Lamberton PH et al., 2014. Onchocerciasis transmission in Ghana: biting and parous rates of host-seeking sibling species of the Simulium damnosum complex. Parasit Vect 7: 511.
8. Toé LD et al., 2014. Optimization of the Esperanza Window Trap for the collection of the African onchocerciasis vector Simulium damnosum sensu lato. Acta Trop 137: 39-43.

9. Rodríguez-Pérez MA et al., 2013. Development of a novel trap for the collection of black flies of the Simulium ochraceum complex. PLoS One 8: e76814.

10. Rodríguez-Pérez MA, Adeleke MA, Rodríguez-Luna IC, Cupp EW, Unnasch TR, 2014. Evaluation of a community-based trapping program to collect Simulium ochraceum sensu lato for verification of onchocerciasis elimination. PLoS Negl Trop Dis 8: e3249.

11. Loum D, Katholi CR, Lakwo T, Habomugisha P, Tukahebwa EM, Unnasch TR, 2017. Evaluation of community-directed operation of black fly traps for entomological surveillance of Onchocerca volvulus transmission in the madi-mid north focus of onchocerciasis in northern Uganda. Am J Trop Med Hyg 97: 1235-1242.

12. Hendy $A$ et al., 2017. Esperanza Window Traps for the collection of anthropophilic black flies (Diptera: Simuliidae) in Uganda and Tanzania. PLoS Negl Trop Dis 11: e0005688.

13. Young RM et al., 2015. Identification of human semiochemicals attractive to the major vectors of onchocerciasis. PLoS Negl Trop Dis 9: e3450.

14. Bernier UR, Kline DL, Schreck CE, Yost RA, Barnard DR, 2002. Chemical analysis of human skin emanations: comparison of volatiles from humans that differ in attraction of Aedes aegypti (Diptera: Culicidae). J Am Mosq Control Assoc 18: 186-195.

15. Walsh JF, 1983. Sampling Simuliid black flies. Youdeowei A, Service MW, eds. Pest and Vector Management in the Tropics. London, United Kingdom: Longman, 93-99.

16. Loum D, Cozart D, Lakwo T, Habomugisha P, Jacob B, Cupp EW, Unnasch TR, 2019. Optimization and evaluation of the Esperanza Window Trap to reduce biting rates of Simulium damnosum sensu lato in northern Uganda PLoS Negl Trop Dis 13: e0007558.

17. Nordstrom KM, McGinley KJ, Cappiello L, Zechman JM, Leyden JJ, 1987. Pitted keratolysis. The role of Micrococcus sedentarius. Arch Dermatol 123: 1320-1325.

18. Singh G, Naik CL, 2005. Pitted keratolysis. Indian J Dermatol Venereol Leprol 71: 213-215.

19. Longshaw CM, Wright JD, Farrell AM, Holland KT, 2002. Kytococcus sedentarius, the organism associated with pitted keratolysis, produces two keratin-degrading enzymes. J Appl Microbiol 93: 810-816.

20. Nordstrom KM, McGinley KJ, Cappiello L, Leyden JJ, 1986. The etiology of the malodor associated with pitted keratolysis. J Invest Dermatol 87: 159.

21. Marshall J, Holland KT, Gribbon EM, 1988. A comparative study of the cutaneous microflora of normal feet with low and high levels of odour. J Appl Bacteriol 65: 61-68.

22. Sanchez JM, Sacks RD, 2006. Development of a multibed sorption trap, comprehensive two-dimensional gas chromatography, and time-of-flight mass spectrometry system for the analysis of volatile organic compounds in human breath. Anal Chem 78: 3046-3054.

23. Birkett MA, Agelopoulos N, Jensen KM, Jespersen JB, Pickett JA, Prijs HJ, Thomas G, Trapman JJ, Wadhams LJ, Woodcock CM, 2004. The role of volatile semiochemicals in mediating host location and selection by nuisance and disease-transmitting cattle flies. Med Vet Entomol 18: 313-322.

24. Magalhaes-Junior JT, Mesquita PR, Oliveira WF, Oliveira FS, Franke CR, Rodrigues Fde M, de Andrade JB, Barrouin-Melo $S M, 2014$. Identification of biomarkers in the hair of dogs: new diagnostic possibilities in the study and control of visceral leishmaniasis. Anal Bioanal Chem 406: 6691-6700.

25. Verocai GG, Mcgaha TW, Jr., Iburg JP, Katholi CR, Cupp EW, Noblet R, Unnasch TR, 2017. Identification of semiochemicals attractive to Simulium vittatum (IS-7). Med Vet Entomol 31: 140-149.

26. Gebrezgabiher G, Mekonnen Z, Yewhalaw D, Hailu A, 2019. Reaching the last mile: main challenges relating to and recommendations to accelerate onchocerciasis elimination in Africa. Infect Dis Poverty 8: 60.

27. Boussinesq M, Fobi G, Kuesel AC, 2018. Altemative treatment strategies to accelerate the elimination of onchocerciasis. Int Health 10: i40-i48.

28. Rodriguez-Perez MA, Garza-Hernandez JA, Salinas-Carmona MC, Femandez-Salas I, Reyes-Villanueva F, Real-Najarro O, Cupp EW, Unnasch TR, 2017. The Esperanza Window Trap reduces the human biting rate of Simulium ochraceum s.l. in formerly onchocerciasis endemic foci in southern Mexico. PLoS Negl Trop Dis 11: e0005686. 